\title{
Маркетинговое исследование рынка мобильных приложений
}

\section{Олеся Ватолина*}

Тихоокеанский государственный университет, Хабаровск, Россия

\author{
Информация о статье \\ Поступила в редакиию: \\ 16.11.2020 \\ Принята \\ к опубликованию: \\ 11.02.2021 \\ УДК 339.138 \\ JEL O14, C12, L96
}

\begin{abstract}
Аннотация
Статья посвящена маркетинговому исследованию рынка мобильных приложений через универсальную платформу - магазин приложений. Основываясь на данных проведенного соичологического опроса, анализе рынка мобильных приложений, а также эффективности инструментов взаимодействия участников рынка разработана адаптация модели уровней товара по Ф. Котлеру, построена схема взаимодействия участников рынка мобильных приложений, проведен математический анализ зависимости выявленных факторов с помощью метода критерия $\chi^{2}$ Пирсона.
\end{abstract}

\section{Mobile App Market Research}

Olesya Vatolina, Dmitry Kolodin

\begin{abstract}
The article presents the main theses of the mobile app market research. The analysis of Russian scientific literature shows shortcomings in the formation of the conceptual framework, marketing concepts, and economic models of interaction between manufacturers and consumers in the field of mobile applications. The conducted studies allow us to conclude that consumers are ready to contribute money for high-quality, in terms of support, content of the mobile applications used. The modern world is characterized by instability and volatility, a high rate of changes occurring, therefore, special increased requirements for the content of relevant information are imposed on mobile applications. The consumer is ready to pay for more popular, unique and necessary in daily use mobile applications, and this creates conditions for the further development of the mobile applications market. The year 2020 showed that the ability to obtain the necessary information, purchase goods and provide services in conditions of physical isolation and social distancing using a convenient means of access is a prerequisite during a pandemic period. Undoubtedly, the market for mobile applications will expand, and the number of participants and services will grow.
\end{abstract}

Keywords:

mobile app, app store, mobile app market, product level model

\section{Ключевые слова:}

зин приложений, рынок мобильных приложений, модель model

* Автор для связи: olvatolina@yandex.ru DOI: https://dx.doi.org/10.24866/2311-2271/2021-1/94-102 
Начало XXI в. характеризуется цифровизацией всех процессов и перманентным ростом объема мирового рынка мобильных приложений. Анализ отечественной научной литературы свидетельствует о недоработках в формировании понятийного аппарата, маркетинговых концепций, экономических моделей взаимодействия производителя в сфере мобильных приложений - разработчика и потребителя - пользователя. В полной мере не определен и не исследован бизнеспроцесс монетизации мобильных приложений на уровне «разработчик» - «потребитель» - «рекламодатель». Проблема коммуникации и обратной связи между данными субъектами еще не стала предметом экономических исследований.

Магазин приложений представляет собой цифровую платформу, на базе которой осуществляется распространение программного обеспечения, в частности мобильных приложений. Под мобильным приложением в данной работе понимается программное обеспечение, которое пользователь приобретает и устанавливает на мобильное устройство. В наиболее популярных магазинах приложений (AppStore, Google Play) скачивание мобильных приложений отождествляется с покупкой, а скачанные на мобильное устройство приложения именуется покупкой. В данном научном исследовании мобильное приложение рассматривается как товар.

Проанализировав и сравнив многоуровневую модель товара В. Благоева [1], мультиатрибутивную модель товара Жан Жака Ломбена [2], многоуровневую модель товара Ф. Котлера [3], был сделан выбор в пользу последней модели, как наиболее полной. Мобильное приложение было рассмотрено через призму модели уровней товара по Ф. Котлеру (рис. 1).

При создании товара разработчику необходимо воспринимать идею на трёх уровнях. Основополагающим является уровень товара по замыслу, на котором даётся ответ на вопрос, что в действительности будет приобретать потребитель.

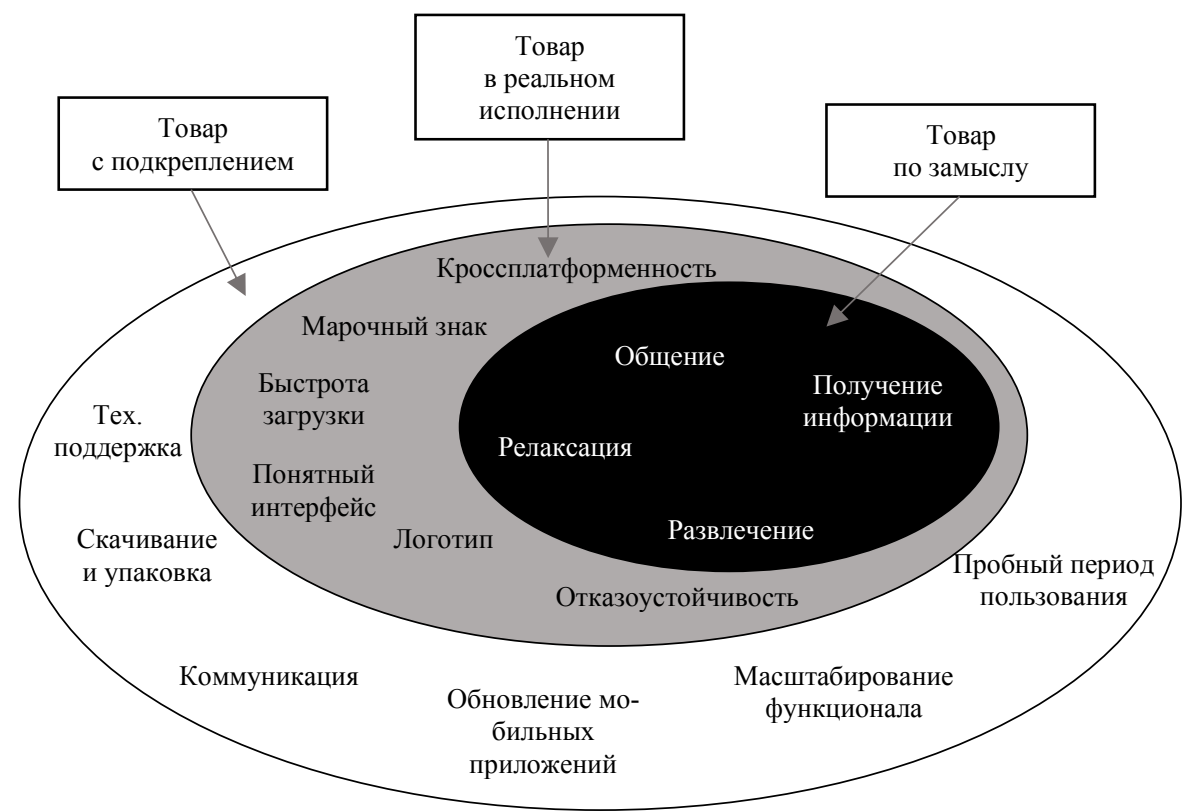

Puc. 1. Адаптация модели товара по Ф. Котлеру к рынку мобильных приложений Источник: составлено авторами 
Авторы определяют содержание уровней товара по Ф. Котлеру относительно мобильного приложения согласно следующей структуре (табл. 1).

Таблица 1

Адаптация модели товара

по Ф. Котлеру к рынку мобильных приложений

\begin{tabular}{|c|c|c|}
\hline Уровень & $\begin{array}{l}\text { Содержание уровня } \\
\text { по Ф. Котлеру }\end{array}$ & $\begin{array}{l}\text { Адаптированная модель } \\
\text { Ф. Котлера к рынку } \\
\text { мобильных приложений }\end{array}$ \\
\hline Товар по замыслу & Основная выгода или услуга & $\begin{array}{l}\text { Общение, релаксация, развлечение, } \\
\text { получение информации. }\end{array}$ \\
\hline \multirow{4}{*}{$\begin{array}{l}\text { Товар в реальном } \\
\text { исполнении }\end{array}$} & Свойства & Быстрота загрузки \\
\hline & Внешнее оформление & Понятный интерфейс \\
\hline & Качество & $\begin{array}{l}\text { Кроссплатформенность, } \\
\text { безотказность }\end{array}$ \\
\hline & Марочное название & Логотип, марочный знак \\
\hline \multirow{4}{*}{$\begin{array}{l}\text { Товар } \\
\text { с подкреплением }\end{array}$} & Гарантийное обслуживание & Техническая поддержка \\
\hline & Послепродажное обслуживание & Обновление \\
\hline & Монтаж & Масштабирование функционала \\
\hline & Поставки и кредитование & $\begin{array}{l}\text { Пробный период } \\
\text { для платных приложений }\end{array}$ \\
\hline
\end{tabular}

Источник: составлено авторами

Ряд авторов, таких как 3. Парк, Б. Яворский, Дж.Шет, Б. Ньюмен, Б. Гросс, Г. Галларза, И. Гил-Сора, Б. Холбрук, Т. Левитт в структуру потребительской ценности товара включаются выгоды, получаемые потребителем [4-7]. Потребительская ценность складывается из различных выгод, получаемых потребителями. Потребительская ценность включает в себя как выгоды, так и затраты потребителей. Сегодня уже не только специалистам, но и широкой общественности очевидно, что рынок мобильных приложений неумолимо расширяется и в нем отчетливо прослеживаются центробежные силы (табл. 2).

Таблица 2

Таблица интересов участников рынка мобильных приложений

\begin{tabular}{|l|l|l|l|}
\hline \multirow{2}{*}{ Участник } & \multicolumn{1}{|c|}{ Песплатное } & \multicolumn{1}{|c|}{ Платное } & \multicolumn{1}{|c|}{ Условно-бесплатное } \\
\cline { 2 - 4 } Разработчик & $\begin{array}{l}\text { Получение прибыли } \\
\text { от рекламодателя }\end{array}$ & $\begin{array}{l}\text { Получение прибыли } \\
\text { от потребителя }\end{array}$ & $\begin{array}{l}\text { Получение прибыли } \\
\text { от потребителя } \\
\text { по истечении } \\
\text { пробного периода }\end{array}$ \\
\hline Потребитель & $\begin{array}{l}\text { Бесплатное } \\
\text { использование } \\
\text { приложения }\end{array}$ & $\begin{array}{l}\text { Использование } \\
\text { приложения } \\
\text { без рекламы }\end{array}$ & $\begin{array}{l}\text { Возможность оценки } \\
\text { платного приложения } \\
\text { до покупки }\end{array}$ \\
\hline $\begin{array}{l}\text { Магазин } \\
\text { приложений }\end{array}$ & $\begin{array}{l}\text { Извлечение прибыли } \\
\text { за показ рекламы }\end{array}$ & $\begin{array}{l}\text { Получение прибыли } \\
\text { за счет комиссии }\end{array}$ & $\begin{array}{l}\text { Получение прибыли } \\
\text { за счет комиссии } \\
\text { по истечении } \\
\text { пробного периода }\end{array}$ \\
\hline Рекламодатель & $\begin{array}{l}\text { Таргетированная } \\
\text { реклама }\end{array}$ & $\begin{array}{l}\text { Таргетированная } \\
\text { реклама во время } \\
\text { пробного периода }\end{array}$ \\
\hline
\end{tabular}

Источник: составлено авторами

Информационные и экономические и потоки, как это видно на схеме (рис. 2) сходятся и контролируются центром схемы - магазином приложений. 


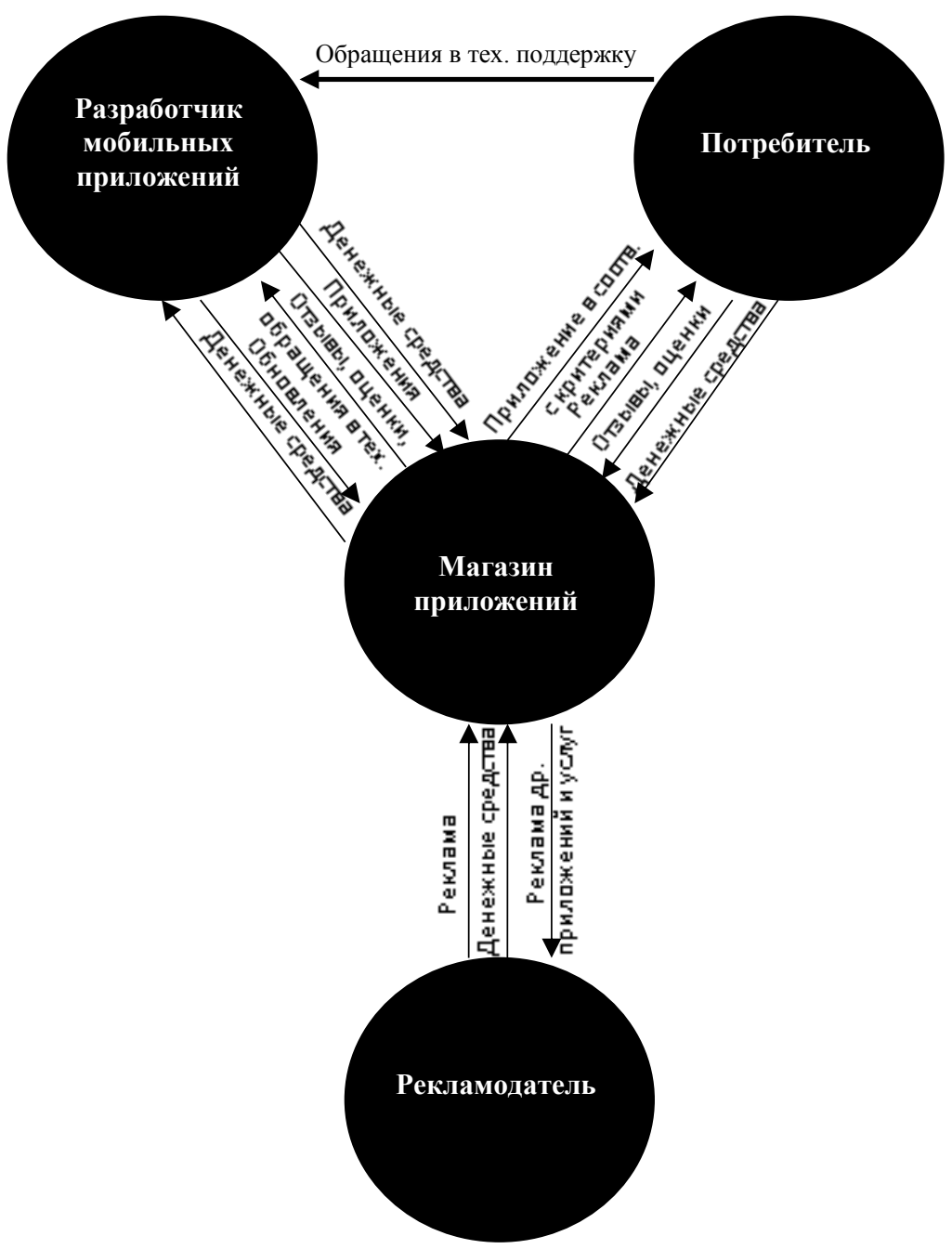

Puc. 2. Схема взаимодействия участников рынка мобильных приложений Источник: составлено авторами

Магазин приложений авторами рассматривается как площадка для оборота денежных средств, распространения и поддержки мобильных приложений, реализации рекламного контента, получения обратной связи, относительно реализованных мобильных приложений, от потребителей.

Разработчик мобильных приложений согласовывает и размещает свой виртуальный продукт в магазин приложений, выплачивает ежегодный взнос за размещение мобильного приложения на площадке. На основании отзывов, оценок, обращений в техническую поддержку разрабатывается обновление мобильных приложений, устраняются сбои работы приложения. При покупке мобильного приложения потребителем и при покупке рекламы в мобильном приложении разработчику поступают денежные средства.

На основании личных предпочтений (рис. 3), Потребитель скачивает и устанавливает мобильное приложение из магазина приложений. 


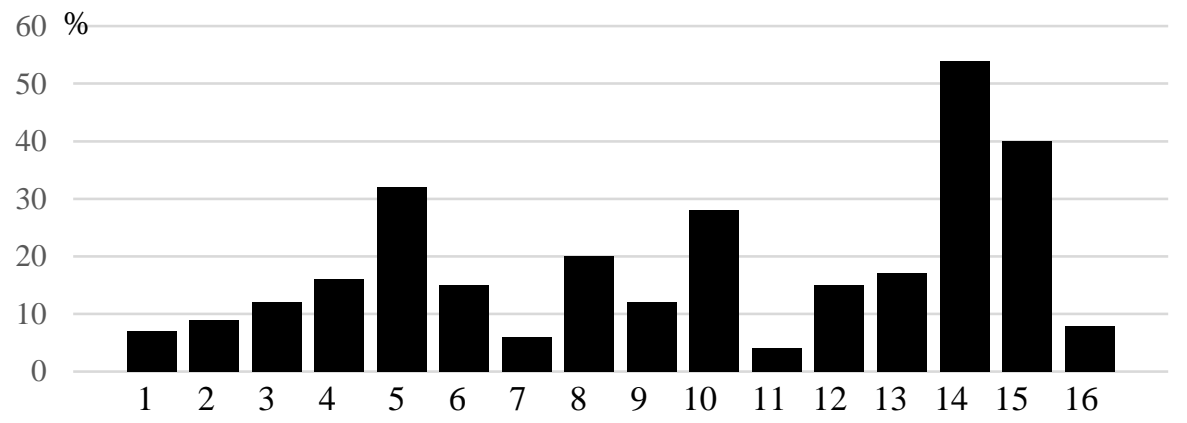

Puc. 3. Категории наиболее привлекательных платных мобильных приложений Примечание: 1 - погода; 2 - новости; 3 - путешествия; 4 - утилиты; 5 - игры; 6 - финансы; 7 - еда; 8 - бизнес; 9 - навигация; 10 - социальные сети; 11 - ритейл (Я - продавец); 12 - интернет-магазины; 13 - здоровье и фитнес; 14 - музыка; 15 - обработка фото и видео; 16 - потоковое видео Источник: составлено авторами

Данные о личных предпочтениях получены на основе эмпирического сбора данных, а именно исследования общественного мнения относительно использования мобильных приложений, отношения к маркетинговым аспектам при использовании мобильных приложений [8]. Исследование проводилось без привязки к социально-демографическим показателям респондентов. В качестве метода было выбрано анкетирование с использованием специально разработанного опросника, состоящего из 18 вопросов, отражающих социальные показатели и отношение респондентов к ряду маркетинговых аспектов рынка мобильных приложений. Анкета была реализована в Google - форме и распространялась через мобильные мессенджеры в виртуальном пространстве и через QR-код на бумажных носителях. На вопросы цифровой анкеты ответили 512 человек, жителей г. Хабаровск различных социально-демографических показателей. По возрасту респонденты распределились следующим образом: до 18 лет - 5,9\%, 18-23 - 57\%, 24-36 $-23,8 \%, 37-45-7,8 \%$, более $45-5,3 \%$. Среди опрошенных были $33,2 \%$ мужчин, 68,8\% женщин [8].

Потребитель может высказать свою удовлетворенность или неудовлетворенность мобильным приложением следующими способами: выставление «оценки», написание отзыва, обращение в техническую поддержку, удаление приложения (неотслеживаемая категория). Если это приложение бесплатное, то в нем систематически появляется встроенная реклама. Если потребителя устраивает соотношение цены мобильного приложения, качества и характера предоставляемой мобильным приложением услуги, он может приобрести подписку этого приложения. В этом случае потребитель получает статус премиум пользователя, что регламентирует расширение функционала приложения или работу приложения в режиме «свободно от рекламы».

Расходы потребителя в магазине приложений включают только затраты, которые проходят через Магазин приложений (платные загрузки, покупки в приложении и подписки в приложении). Это не включает транзакции, обработанные за пределами Магазинов приложений (например, когда приложение выступает посредником в получении услуги).

Рекламодатель приобретает за денежные средства в Магазине приложений показ рекламы, «клики» по виртуальным рекламным баннерам, переходы потребителей на требуемый ресурс. В ряде случаев Разработчик мобильных приложений является Рекламодателем. Рекламодатель использует разнообразие 
мобильных приложений для показа рекламы, тем самым увеличивает охват, находят более выгодные цены, а также верифицирует аудиторию, для оптимизации таргетирования.

Магазин приложений верифицирует мобильное приложение по описанию, функционалу, дизайну, безопасности потребителей, безотказности, содержанию, соответствию законодательству стран, в которых данное мобильное приложение доступно.

В начале эмпирического исследования была поставлена гипотеза о связи между социальными и экономическими факторами при взаимодействии потребителя и разработчика. После получения данных социологического опроса, было решено верифицировать корреляционную зависимость с помощью метода критерия $\chi^{2}$ Пирсона [9]. Критерий $\chi^{2}$ Пирсона позволил оценить значимость различий между фактическим (выявленным в результате исследования) количеством исходов или качественных характеристик выборки, попадающих в каждую категорию (табл. 3), и теоретическим количеством, которое можно ожидать в изучаемых группах при справедливости нулевой гипотезы (табл. 4). Была определена значимость влияния уровня образования на готовность вносить плату за мобильное приложение, которое респондент использует ежедневно, если оно станет платным.

Таблица 3

Эмпирическое разделение представленных признаков

\begin{tabular}{|l|c|c|c|c|}
\hline \multirow{2}{*}{ Уровень образования } & \multicolumn{4}{|c|}{ Варианты ответа } \\
\cline { 2 - 5 } & Да & Неm & Зависит от стоимости & $\sum_{\boldsymbol{j}} \boldsymbol{n}_{\boldsymbol{i}, \boldsymbol{j}}$ \\
\hline Высшее & 28 & 59 & 95 & 182 \\
\hline Незаконченное высшее & 59 & 45 & 106 & 210 \\
\hline Среднее специальное & 7 & 24 & 38 & 69 \\
\hline Среднее & 7 & 19 & 25 & 512 \\
\hline$\sum_{i} n_{i, j}$ & 101 & 147 & 264 & 512 \\
\hline
\end{tabular}

Источник: составлено авторами

Расчёт теоретического распределения признаков осуществлялся по формуле:

$$
f_{i}^{\prime}=\frac{n_{i} * n_{j}}{n}
$$

Теоретическое распределение представленных признаков

\begin{tabular}{|l|c|c|c|c|}
\hline \multirow{2}{*}{ Уровень образования } & \multicolumn{4}{|c|}{ Варианты ответа } \\
\cline { 2 - 5 } & \multirow{2}{*}{$\boldsymbol{H}$} & $\boldsymbol{H e m}$ & Зависит от стоимости & $\sum_{\boldsymbol{j}} \boldsymbol{n}_{\boldsymbol{i}, \boldsymbol{j}}$ \\
\hline Высшее & 35,90 & 52,25 & 93,84 & 182 \\
\hline Незаконченное высшее & 41,43 & 60,29 & 108,28 & 210 \\
\hline Среднее специальное & 13,61 & 19,81 & 35,58 & 69 \\
\hline Среднее & 10,06 & 14,64 & 26,30 & 51 \\
\hline$\sum_{i} n_{i, j}$ & 101 & 147 & 264 & 512 \\
\hline
\end{tabular}

Источник: составлено авторами

Расчет значения критерия $\chi^{2}$ Пирсона осуществляется по формуле 


$$
\mathrm{x}_{\text {эмп }}^{2}=\sum_{i} \frac{\left(f_{i}-f_{i}^{\prime}\right)^{2}}{f_{i}^{\prime}}
$$

В данном случает число степеней свободы рассчитывается следующим образом $\mathrm{k}=(4-1)^{*}(3-1)=6$. Критическая точка $\chi^{2}$ при уровне значимости 0,05 и числе степеней свободы $\mathrm{k}=6$ находим критическую точку $\mathrm{x}_{\text {крит }}^{2}(0,05,6)=12,6$.

Было проведено сравнение полученного значения критерия $\chi^{2}$ с критиче-

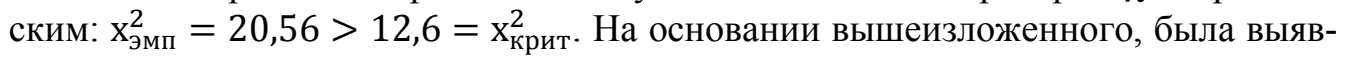
лена зависимость частоты случаев готовности платить за мобильное приложение, от фактора «уровень образования» - связь подвержена. Аналогично были проверены взаимосвязи других факторов исследования (табл. 5).

Таблица 5

Выявление связи между факторами на основе критерия $x^{2}$ Пирсона

\begin{tabular}{|c|c|c|c|}
\hline Фактор 1 & Фактор 2 & $\begin{array}{c}\text { Фактическое } \\
\text { значение } \\
\text { критерия } \\
\text { Пирсона } \\
\end{array}$ & $\begin{array}{l}\text { Подтверждение/ опро- } \\
\text { вержение связи }\end{array}$ \\
\hline Уровень образования & $\begin{array}{l}\text { Периодичность установки платных } \\
\text { мобильных приложений }\end{array}$ & 10,62 & Связь отсутствует \\
\hline Уровень образования & $\begin{array}{l}\text { Важность регулярного обновления } \\
\text { установленных бесплатных мобиль- } \\
\text { ных приложений }\end{array}$ & 3,3 & Связь отсутствует \\
\hline Уровень образования & $\begin{array}{l}\text { Важность регулярного обновления } \\
\text { установленных платных мобильных } \\
\text { приложений }\end{array}$ & 17,48 & Связь подтверждается \\
\hline $\begin{array}{l}\text { Готовность платить } \\
\text { за дополнительные } \\
\text { услуги в условно-бес- } \\
\text { платном мобильном } \\
\text { приложении }\end{array}$ & $\begin{array}{l}\text { Важность регулярного обновления } \\
\text { установленных бесплатных мобиль- } \\
\text { ных приложений }\end{array}$ & 6,1 & Связь подтверждается \\
\hline Уровень образования & $\begin{array}{l}\text { Готовы ли Вы платить за мобильное } \\
\text { приложение, которое используете } \\
\text { ежедневно, если оно станет платным }\end{array}$ & 20,56 & Связь подтверждается \\
\hline
\end{tabular}

Источник: составлено авторами

На основе критерия $\chi^{2}$ Пирсона были сделаны выводы о наличии статистической взаимосвязи, подтверждена гипотеза о корреляции между рассмотренными факторами при соответствующем уровне значимости. Исследование подтверждает, что для людей с высшим образованием, или получаемым высшим образованием важным фактором для приобретения мобильного приложения является регулярное обновление или «послепродажное обслуживание» по Ф. Котлеру. Также респонденты с той же социальной характеристикой демонстрируют готовность вносить денежные средства за бесплатное мобильное приложение, которое используют ежедневно, если оно станет платным. Помимо вышесказанного, была установлена связь, между ответами респондентов, о готовности платить за дополнительные услуги в «условно-бесплатном» мобильном приложении и важности регулярного обновления установленных бесплатных мобильных приложений.

Проведенные исследования позволяют сделать вывод о готовности потребителей вносить денежные средства за качественный, с точки зрения поддержки, контент используемых мобильных приложений.

Рынок мобильных приложений имеет годовой доход в миллиарды долларов, не производя материальный товар. Три крупнейших компании, работающей 
с фокусом на мобильные приложения, обгоняют многие корпорации, ориентированные на материальный товар: Spotify (онлайн музыка) (29,5 млрд долларов), Pinduoduo (ритейл - сервис) (24 млрд долларов) и Tencent Music Entertainment (потоковый музыкальный контент) (21,3 млрд долларов) [10].

Современный мир характеризуется нестабильностью и изменчивостью, высокой скоростью происходящих изменений, поэтому к мобильным приложениям предъявляется особые повышенные требования к содержанию актуальной информации. За более востребованные, уникальные и необходимые в ежедневном использовании мобильные приложения потребитель готов платить, и это создает условия для дальнейшего развития рынка мобильных приложений. 2020 год показал, что возможность получения необходимой информации, приобретения товаров и оказания услуг в условиях физического изоляции и социального дистанцирования с использованием удобного средства доступа является необходимым условием в период пандемии. Несомненно, рынок мобильных приложений будет расширяться, а количество участников и услуг будет расти.

\section{Список источников / References}

1. Благоев В. Маркетинг в определениях и примерах. - СПб.: «Два ТрИ», 1999. - С.251. [Blagoev V. Marketing v opredeleniyakh i primerakh. - SPb.: «Dva TrI», 1999. - S.251.]

2. Ламбен Ж.Ж. Стратегический маркетинг. Европейская перспектива. - М.: Наука, 2004. - C.98-121. [Lamben Zh.Zh. Strategicheskiy marketing. Evropeyskaya perspektiva. - M.: Nauka, 2004. - S.98-121.]

3. Котлер Ф. Основы маркетинга: пер с англ. - 2 -е европ. изд. Москва, Издательский дом «Вильямс», 2002. 944 с. [Kotler F. Osnovy marketinga : Persangl. - 2- e evrop. izd. [Marketing Basics]. Moscow, Williams Publ., 2002. 944 p.]

4. Park C.W., Jaworski B.J., MacInnis D.J. Strategic brand concept - image management. The Journal of Marketing, 1986. pp. 135- 145.

5. Sheth J.N., Newmen B.I, Gross B.l. Consumption Values and Market Choices: Theory and Applications, Cincinaty, OH: South Western Publishing Company, 1991.

6. Gallarza G.M., Gil-Saura I., Holbrook M.B. The value of value: Furthe excursions on the meaning and role of customer value Journal of Consumer Behaviour, J. Consumer Behav , 2011, no. 10, pp. 179 - 191.

7. Levitt T. Marketing Success through Differentiation - of Anything, Harvard Business Review, January - February 1980.

8. Колодин Д. В., Ватолина О. В. Коммуникационный инструментарий социального взаимодействия в информационном обществе: мобильные приложения // Власть и управление на Востоке России. 2020. № 3 (92) [Kolodin D. V., Vatolina O. V. Kommunikacionnyj instrumentarij social'nogo vzaimodejstvija $v$ informacionnom obshhestve: mobil'nye prilozhenija // Vlast' i upravlenie na Vostoke Rossii. 2020. № 3 (92)].

9. Кендалл М., Стьюарт А. Статистические выводы и связи. - М.: Наука, 1973 [Kendall M., St'yuart A. Statisticheskie vyvody i svyazi. — M.: Nauka, 1973].

10. Отчёт App Annie: весь рынок мобильных приложений за 2018 год [Otchiot App Annie: ves' rynok mobil'nykh prilozheniy za 2018 god]. Available at: https://zen.yandex.ru/media/id/5bee94ac7c03d700aa4e5464/otchet-app-annie-ves-rynok-mobilnyh-prilojenii-za2018-god-5c49c6ea3bf2f400af71ce84 (accessed 10.06.2020). 


\section{Сведения об авторе / About author}

Ватолина Олеся Владимировна, кандидат экономических наук, доцент кафедры «Экономическая кибернетика», Тихоокеанский государственный университет. 680000 Россия, г. Хабаровск, ул. Тихоокеанская, д. 136. E-mail: olvatolina@yandex.ru

Olesya V. Vatolina, Candidate of Economics Sciences, Associate Professor of the Department of Economic Cybernetics, Pacific State University. 136, Tikhookeanskay str., Khabarovsk, Russia 680000.

E-mail: olvatolina@yandex.ru

Колодин Дмитрий Владимирович, кандидат социологических наук, заместитель директора Департамента организации образовательной деятельности, Дальневосточный федеральный университет. 690022 Россия, г. Владивосток, о-в Русский, кампус ДВФУ; магистрант кафедры «Экономическая кибернетика» направление 09.04.03 Прикладная информатика магистерская программа «Прикладная информатика в экономике», Тихоокеанский государственный университет. 680000 Россия, г. Хабаровск, ул. Тихоокеанская, д. 136. E-mail: dmitrii.kolodin@yandex.ru

Dmitriy V. Kolodin, Candidate of Sociology Sciences, Deputy Director of the Department for Organization of Educational Activities, Far Eastern Federal University. FEFU campus, Russky Island, Vladivostok, Russia 690922. Master Student of the Department of Economic Cybernetics, direction 09.04.03 Applied Informatics Master's program "Applied Informatics in Economics", Pacific State University. 136, Tikhookeanskay str., Khabarovsk, Russia 680000. E-mail: dmitrii.kolodin@yandex.ru 